\title{
How much shorter is better? Investigating image acquisition time reduction on left ventricular phase analysis for cardiac dyssynchrony
}

\author{
John O. Prior, PhD, $M^{\mathrm{a}}$ \\ a Department of Nuclear Medicine and Molecular Imaging, Lausanne University Hospital, \\ Lausanne, Switzerland
}

Received May 31, 2015; accepted Jun 1, 2015

doi: 10.1007/s12350-015-0196-2

See related article, pp. 643-651

\section{EDITORIAL}

Myocardial perfusion imaging is a widespread technique with proven added value for CAD diagnosis, prognostication, and therapeutic monitoring. ${ }^{1}$ In the last decade, there have been many improvements in gammacamera hardware, software reconstructions algorithms including resolution recovery methods, and cardiac evaluation software. ${ }^{2,3}$ This has led to shortening of the acquisition time as compared to previous guidelines, going from 30 seconds down to 15 seconds per projection (the so-called "half-time" acquisition). ${ }^{4}$ Gating the acquisition with ECG has the ability to measure cardiac wall motion and thickening and left ventricular systolic and diastolic volumes, as well as ejection fraction (EF). The shortening of the acquisition time brings advantages in terms of images degradation due to patient movement, upwards heart creep in poststress period, patients throughput, as well as in patients comfort at the expense of perfusion defects detectability due to increase in image noise. ${ }^{5}$

A developing application of gated-SPECT MPI has been the investigation of left ventricular dyssynchrony using the phase analysis technique. ${ }^{6-10}$ This method

Reprint requests: John O. Prior, PhD MD, Department of Nuclear Medicine and Molecular Imaging, Lausanne University Hospital, Rue du Bugnon 46, 1011, Lausanne, Switzerland; john.prior@ chuv.ch

J Nucl Cardiol 2015;22:652-4.

$1071-3581 / \$ 34.00$

Copyright (c) 2015 American Society of Nuclear Cardiology. delivers unique information about the intrinsic contractile ventricular properties, which can help deciding on which to refer patients to resynchronization therapy, ${ }^{11,12}$ as well as adding prognostication. ${ }^{13}$ There has not been any investigation published on examining the effect of SPECT acquisition time reduction on phase analysis. This is exactly the aim of the work by Kortelainen and co-authors published in this issue. ${ }^{14}$ The authors investigated the relation of left ventricular functional parameters and phase histogram to acquisition time reduction in a population of 24 patients referred for stress/rest gated-SPECT MPI. Among these patients, 20 $(83 \%)$ had at least some slight perfusion abnormalities at rest and $9(37.5 \%)$ patients had previous cardiac infarct or heart failure. Methodologically, the authors used listmode acquisitions and recording of the ECG to mimic shorter acquisition times $(80 \%, 60 \%, 50 \%, 40 \%, 30 \%$, $20 \%$ ) than the initial 30 seconds per projection (100\%).

In a nutshell, the authors found that reducing acquisition time from 30 seconds down to 15 seconds had no clinically significant effect in left ventricular EF (Figure 1), wall motion, or wall thickening. When decreasing acquisition time further, statistically lower values were noted in end diastolic volume (EDV) and stroke volume (SV). Interestingly, end systolic volume (ESV) and EF were not affected (Figure 1). In contrary, contrast-to-noise ratio (CNR) already presented significant differences when acquisition time was reduced down to $60 \%$ (18 seconds per projection), with differences at half-time acquisition reaching $-15 \%$ and $-11 \%$ for systolic and diastolic CNR, respectively. Moreover, it was the same with phase analysis, where already statistically significant differences were encountered when the acquisition duration was only decreased to $80 \%$ (phase histogram bandwidth, BW), or $60 \%$ (phase histogram standard deviation, StDev and entropy, ENT), with already large errors at half-time acquisition 


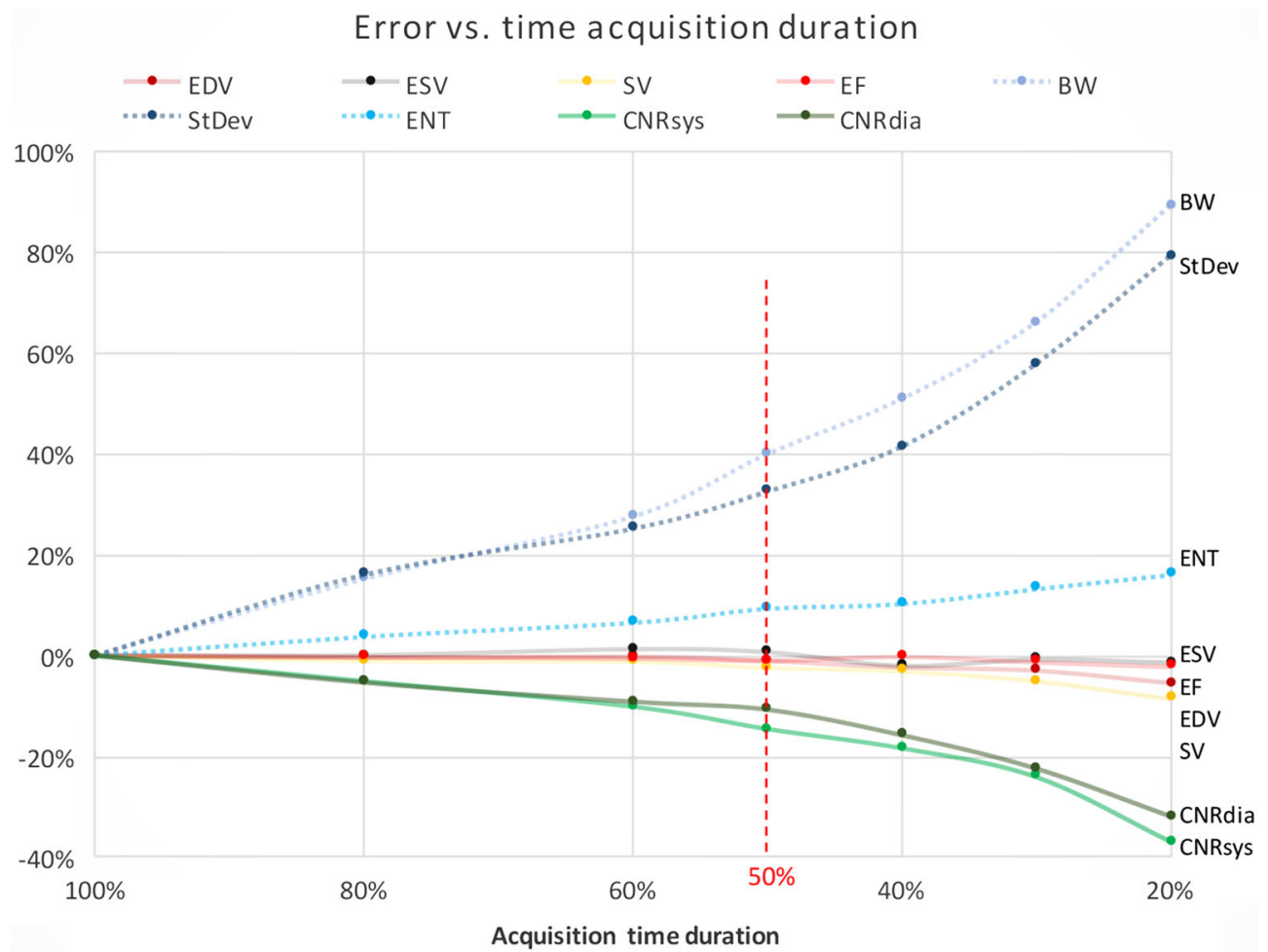

Figure 1. Representation of the error compared to the full $(100 \%)$ acquisition time in relation to varying acquisition time duration in the study by Kortelainen et al. ${ }^{14}$ The horizontal axis represents the time duration in percentage of the full, normal acquisition duration ( 30 seconds per projection). Conventional LV function parameters (ESV, end systolic volume; EF, ejection fraction; EDV, end diastolic volume; SV, stroke volume), as well as wall motion and wall thickening (both not presented on this graphics and $<6 \%$ ) were not significantly affected by acquisition time reduction up to half-time acquisition (50\%). This is contrast to phase analysis, where main parameters differed already significantly for acquisition time less or equal to $80 \%$ (BW $=$ phase histogram bandwidth) or $60 \%$ (StDev $=$ phase histogram standard deviation, ENT $=$ phase histogram entropy), similarly to contrast-to-noise ratio $(\mathrm{CNRdia}=$ contrast-to-noise ratio at diastole, CNRsys $=$ contrast-to-noise ratio at systole).

( $+40 \%$ for phase histogram $\mathrm{BW} ;+32 \%$ for phase histogram standard deviation; $9 \%$ for phase histogram entropy), as illustrated in Figure 1. Accordingly, if phase analysis is to be used for clinical decisions, halftime acquisitions may introduce errors on phase analysis indices and may not be equivalent to full-time acquisitions. This is the important finding of this work.

Of course, several study limitations existed, but the authors ${ }^{14}$ correctly addressed them. One could add that: (i) this work was performed in a population that may not reflect the true population of patients normally referred for cardiac dyssynchrony studies; and (ii) it had been performed using only one specific commercially-available software package. Whether these results would hold in the population of patients referred for cardiac dyssynchrony characterization and if identical results would be obtained with a different software package are still open questions.
The present study by Kortelainen et $\mathrm{al}^{14}$ has the merit of asking a legitimate question and providing an answer. Future trials can already integrate this knowledge in their design, as well as more specifically addressing the effect of reducing acquisition time in patient populations needing cardiac dyssynchrony analysis or in relation to other phase analysis software packages.

\section{Disclosure}

John O. Prior has no conflict of interest to declare.

\section{References}

1. Travin MI. Cardiac radionuclide imaging to assess patients with heart failure. Semin Nucl Med 2014;44:294-313. 
2. Slomka PJ, Berman DS, Germano G. New cardiac cameras: Single-photon emission CT and PET. Semin Nucl Med 2014;44:23251.

3. Gordon DePuey E. Advances in cardiac processing software. Semin Nucl Med 2014;44:252-73.

4. DePuey EG, Gadiraju R, Clark J, Thompson L, Anstett F, Shwartz SC. Ordered subset expectation maximization and wide beam reconstruction "half-time" gated myocardial perfusion SPECT functional imaging: A comparison to "full-time" filtered backprojection. J Nucl Cardiol 2008;15:547-63.

5. Enevoldsen LH, Menashi CA, Andersen UB, Jensen LT, Henriksen OM. Effects of acquisition time and reconstruction algorithm on image quality, quantitative parameters, and clinical interpretation of myocardial perfusion imaging. J Nucl Cardiol 2013;20: 1086-92.

6. Chen J, Garcia EV, Folks RD, Cooke CD, Faber TL, Tauxe EL, et al. Onset of left ventricular mechanical contraction as determined by phase analysis of ECG-gated myocardial perfusion SPECT imaging: Development of a diagnostic tool for assessment of cardiac mechanical dyssynchrony. J Nucl Cardiol 2005;12:687-95.

7. Chen J, Bax JJ, Henneman MM, Boogers MJ, Garcia EV. Is nuclear imaging a viable alternative technique to assess dyssynchrony? Europace 2008;10(Suppl 3):iii101-5.

8. Chen J, Garcia EV, Bax JJ, Iskandrian AE, Borges-Neto S, Soman P. SPECT myocardial perfusion imaging for the assessment of left ventricular mechanical dyssynchrony. J Nucl Cardiol 2011;18: 685-94.
9. Matsuo S. Phase analysis using gated myocardial perfusion singlephoton emission computed tomography imaging for evaluating cardiac dyssynchrony. Circ J 2012;76:1832-3.

10. Igarashi Y, Chikamori T, Hida S, Tanaka H, Shiba C, Usui Y, et al. Usefulness of phase analysis to differentiate ischemic and non-ischemic etiologies of left ventricular systolic dysfunction in patients with heart failure. Circ J 2014;78:141-50.

11. Chen J, Boogers MJ, Bax JJ, Soman P, Garcia EV. The use of nuclear imaging for cardiac resynchronization therapy. Curr Cardiol Rep 2010;12:185-91.

12. Uebleis C, Hellweger S, Laubender RP, Becker A, Sohn HY, Lehner S, et al. Left ventricular dyssynchrony assessed by gated SPECT phase analysis is an independent predictor of death in patients with advanced coronary artery disease and reduced left ventricular function not undergoing cardiac resynchronization therapy. Eur J Nucl Med Mol Imaging 2012;39:1561-9.

13. Goldberg AS, Alraies MC, Cerqueira MD, Jaber WA, Aljaroudi WA. Prognostic value of left ventricular mechanical dyssynchrony by phase analysis in patients with non-ischemic cardiomyopathy with ejection fraction $35-50 \%$ and QRS $<150 \mathrm{~ms}$. J Nucl Cardiol 2014;21:57-66.

14. Kortelainen M, Koivumäki T, Vauhkonen M, Hakulinen M. Dependence of left ventricular functional parameters on image acquisition time in cardiac-gated myocardial perfusion SPECT. J Nuc Cardiol 2015. doi:10.1007/s12350-015-0178-4. 